\title{
Cryptococcosis in patients with hematological diseases: a 14-year retrospective clinical analysis in a Chinese tertiary hospital
}

\author{
Rui-ying Wang ${ }^{\dagger}$, Yan-qiong Chen ${ }^{\dagger}$, Ji-qin Wu, Xuan Wang, Ya-hui Cao, Hua-zhen Zhao and Li-ping Zhu*
}

\begin{abstract}
Background: Cryptococcal infection has become a public health challenge globally. However, information about cryptococcal infection in patients with hematological diseases remains relatively rare.

Methods: HIV-uninfected cryptococcosis cases with hematological diseases admitted to Huashan Hospital from January 2001 to December 2014 were reviewed.

Results: In total, 33 cryptococcosis patients were enrolled, including 12 malignant and 21 non-malignant hematological cases. Twenty-six patients had central nervous system (CNS) involvement, which was observed more often in patients with non-malignancies than with malignancies (20/21 vs. $6 / 12, P=0.001)$ Most patients $(25 / 26)$ with CNS infection were confirmed by cerebrospinal fluid (CSF) culture or smear, and 100\% (20/20) of them tested positive for the CSF cryptococcal antigen test. Eighteen out of 26 cryptococcal meningitis patients were treated with amphotericin B (AmB)-based therapy, 16 of them with AmB deoxycholate ( $d$-AmB) and 2 patients with liposomal AmB. The clinical success rate was $55.6 \%$. D-AmB was well-tolerated at $0.35-0.59 \mathrm{mg} / \mathrm{kg} / \mathrm{d}$ (median 0 . $43 \mathrm{mg} / \mathrm{kg} / \mathrm{d}$ ) and only 12 patients had mild adverse events.

Conclusions: CNS cryptococcal infection was more frequent in patients with hematological non-malignancies, and cryptococcal antigen test as well as the CSF fungal culture or smear are suggested for early diagnosis. D-AmB could be used as an alternative therapy for CNS-infected patients with hematological diseases.
\end{abstract}

Keywords: Cryptococcosis, Hematological diseases, Cryptococcal meningitis, Pulmonary cryptococcosis, HIV-uninfected

\section{Background}

Cryptococcosis is a life threatening disease in patients with immunocompromised conditions, such as human immunodeficiency virus (HIV) infection, solid organ transplants (SOT), autoimmune diseases, administration of corticosteroids and other immunosuppressants $[1,2]$. Globally, about 957,900 cryptococcal meningitis cases occur each year, resulting in 624,700 deaths by 3 months after infection among HIV-infected patients [3]. Moreover, cryptococcal infection could also occur in $2.8-8.0 \%$ of SOT recipients, and is the third most common invasive

\footnotetext{
* Correspondence: zhulp@fudan.edu.cn

${ }^{\dagger}$ Equal contributors

Department of Infectious Diseases, Huashan Hospital, Fudan University, 12 Central Urumqi Road, Shanghai, China
}

fungal infection in these patients, after Candida and Aspergillus infections [4, 5]. However, studies on cryptococcal infections under hematological conditions are rare [6-8]. The SEIFEM-2004 study showed that only 8 cryptococcosis cases were documented among 11,802 patients with hematological malignancies, corresponding to an extremely low incidence of $0.07 \%$ [9]. According to a 30 -year autopsy study, 340 cases had invasive mycoses among 1591 autopsied patients with hematological neoplasias [10]. Among them, Aspergillus and Candida accounted for $52.6 \%$ and $31.4 \%$ respectively, while only 2 patients were confirmed to have cryptococcal infection [10]. In addition, detailed studies of cryptococcosis patients with other non-malignant hematological diseases, such as autoimmune hemolytic anemia (AIHA), immune 
thrombocytopenia (ITP), and Evans' syndrome remain sparse [11]. Here, we retrospectively reviewed a series of cryptococcosis cases with hematological diseases admitted to our hospital from 2001 through 2014 to evaluate the possible risk factors, clinical characteristics, and prognosis of cryptococcosis in this population.

\section{Methods}

\section{Patients and data resources}

We retrospectively reviewed all proven and probable cases of cryptococcosis with hematological diseases admitted to Huashan Hospital, Fudan University (a tertiary health care center in Shanghai, China, with approximately 1200 hospital beds and 60,000 admissions per year) from January 2001 through December 2014. Patient demographics and other medical data were retrieved from the electronic medical record system in Huashan Hospital. Cases of cryptococcosis were identified using International Classification of Diseases (ICD) diagnostic codes. Individuals with one or more ICD-10 codes starting with 'B45' were classified as cryptococcosis cases. Medical records of these cases were carefully checked and patients were finally included in the cohort if a diagnosis of hematological diseases coexisted. None of the patients were HIV-infected. Clinical data of the enrolled patients was collected, including demographic information, underlying hematological diseases, other predisposing factors, clinical characteristics of cryptococcosis, and antifungal treatment.

\section{Definition of cryptococcosis}

A proven diagnosis of cryptococcal meningitis was made if the patient met any of the following criteria: (1) positive culture of Cryptococcus from the cerebrospinal fluid (CSF), (2) positive CSF India ink smear, (3) positive cryptococcal capsular polysaccharide antigen in the CSF or (4) compatible histopathological findings, which comprise 5-10 $\mu \mathrm{m}$ encapsulated yeasts observed in the brain tissue. A proven diagnosis of pulmonary or sinus cryptococcosis was made through histopathology or tissue culture. A probable diagnosis of pulmonary or sinus cryptococcosis was made if all of the following conditions were met: (1) positive cryptococcal antigen titer in blood, (2) lesions of lung or sinus on computerized tomography (CT) scan, and (3) improvement of symptoms and radiology after antifungal treatment. Blood stream infection was confirmed if the blood culture of Cryptococcus was positive. Cryptococcal capsular polysaccharide antigen test of the CSF or serum was conducted using the latex-Cryptococcus antigen detection system (Immuno-Mycologics, Inc., USA). A result of at least 1:10 was considered positive $[12,13]$.

\section{Efficacy and safety assessment of antifungal therapy} Physicians chose antifungal therapy for the patients according to the Chinese guideline for cryptococcosis and available expert recommendations $[14,15]$. Response to antifungal treatment in cryptococcosis patients was evaluated at the end of initial therapy on the basis of clinical, radiologic, and microbiologic data according to the consensus criteria issued by the European Organization for Research and Treatment of Cancer and Mycoses Study Group (EORTC/MSG) [16]. Efficacy of antifungal treatment was categorized as success (i.e., complete or partial response) or failure (i.e., stable response, disease progression, or death during the study period regardless of any cause). Patients with antifungal treatment less than 7 days were not included for efficacy evaluation. All-cause mortality at 2 weeks, 10 weeks and survival at 1-year follow-up were also analyzed. Adverse drug events (ADEs) of antifungal treatment were recorded. Relationship between ADEs and antifungal drugs was evaluated on the basis of the causality assessment system proposed by the World Health Organization Collaborating Centre for International Drug Monitoring, the Uppsala Monitoring Centre (WHO-UMC) [17].

\section{Statistical analysis}

Summary statistics are expressed as mean \pm standard deviations for continuous variables with normal distributions. Other data are expressed as median and range. Continuous variables within two groups were compared using the independent t-test for parametric data and the MannWhitney $U$ test for non-parametric data. Categorical variables were expressed as proportions and compared using the Chi-square test or Fischer's exact test, as appropriate. A $P$-value $<0.05$ was considered statistically significant. Statistical analysis was performed with the SPSS statistical package version 17.0 (SPSS, Inc., Chicago, USA).

\section{Results}

\section{Demographic and clinical characteristics}

During the study period, we identified 33 cryptococcosis patients with hematological diseases, among which 30 were proven and 3 were probable cases. Distribution of the 33 cases over 14 years is shown in Fig. 1. Demographic data and clinical characteristics of each patient were summarized in Table 1, and detailed information is listed in the Additional file 1 . The median age of patients was 50 years (range, 16-79), and 42.4\% were male. Twenty-one patients $(63.6 \%)$ were diagnosed with non-malignant hematological diseases, including AIHA, Evans' syndrome, and ITP. Twelve patients (36.4\%) had confirmed hematological malignancies, including non-Hodgkin's lymphoma (NHL), Waldenstrom's macroglobulinemia (WM), acute lymphocytic leukemia (ALL), chronic lymphocytic leukemia (CLL), Hodgkin's lymphoma (HL), multiple myeloma (MM), and myelodysplastic syndrome (MDS). The median time from the diagnosis of hematological diseases to the time of cryptococcosis was 73 weeks (ranging from 8 weeks to 


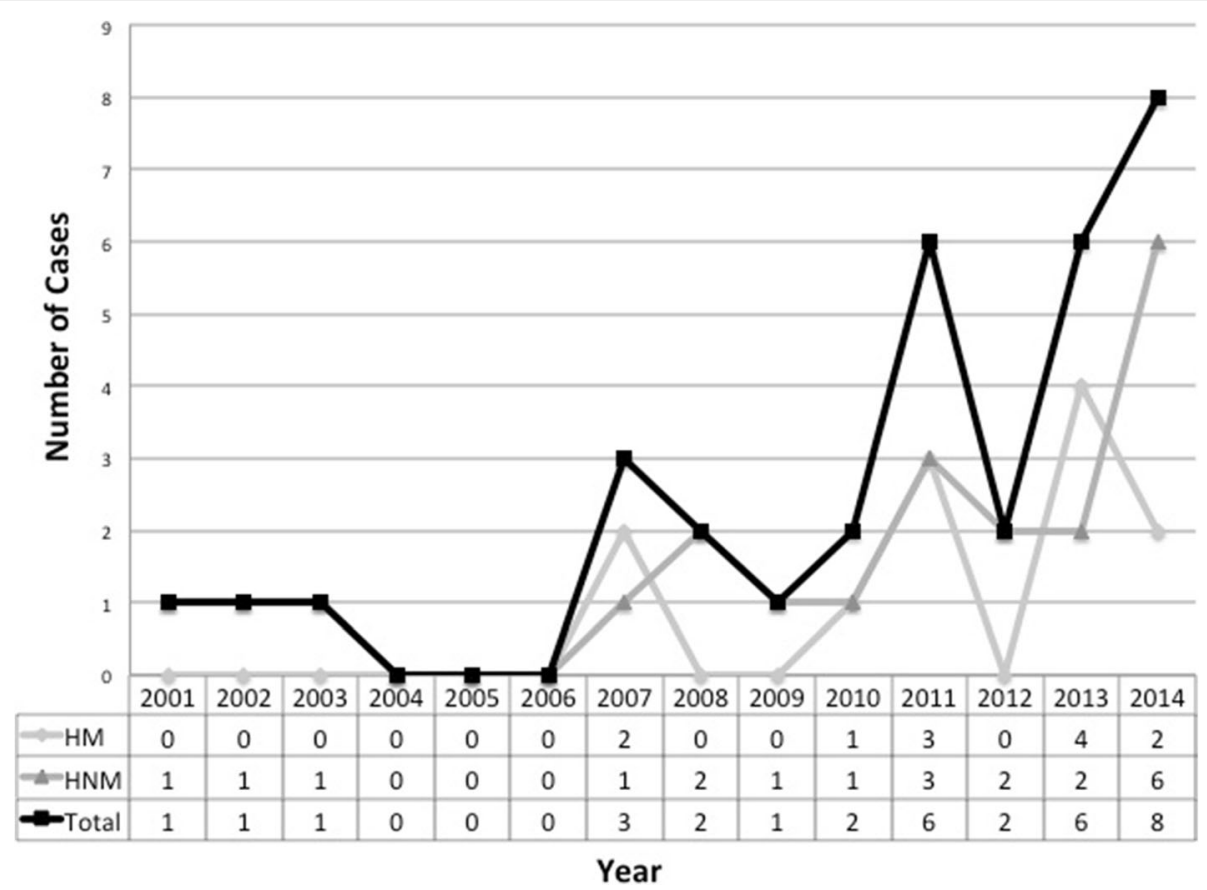

Fig. 1 Distribution of cryptococcosis cases over 14 years. HM = hematological malignancies, HNM = hematological non-malignancies

15 years), except in 4 patients whose hematological diagnosis was confirmed after the onset of cryptococcosis. One patient was diagnosed with NHL 5 months after pulmonary cryptococcosis, one was diagnosed with WM 8 months after pulmonary cryptococcosis, another patient was diagnosed with AIHA 6 weeks after cryptococcal meningitis, and the last was diagnosed with NHL 3 months after cryptococcal meningitis diagnosis. Glucocorticoid usage was documented in $20(20 / 21,95.2 \%)$ of the non-malignancy patients. Usage of prednisone dosage higher than $0.3 \mathrm{mg} / \mathrm{kg} / \mathrm{d}$ for more than 3 weeks was documented in 12 patients. A long-term use of low dose glucocorticoids was documented in 8 patients, and the duration of glucocorticoids use ranged from 2 months to more than 10 years. Eight among the 12 malignancy patients had undergone chemotherapy before infection. Prednisone was included in all chemotherapy regimens. Rituximab was used in 4 patients; among them one also received bevacizumab. Other predisposing factors for cryptococcosis included chronic kidney diseases in 2 , splenectomy in 2, and rectal cancer in 1 patient.

\section{Clinical manifestations}

The sites of cryptococcal infections in patients with hematological diseases included brain in 26 , lung in 16 , blood in 3, and sinus in 2 patients. Some of the patients had multi-site infection. The main clinical manifestations among patients with central nervous system (CNS) infection were fever and headache (both were $24 / 26,92.3 \%$ ), as shown in Table 2. Sixteen of the febrile patients had temperatures higher than $39^{\circ} \mathrm{C}, 5$ patients were between
38.5-39 ${ }^{\circ} \mathrm{C}$, and one patient had a maximum temperature of $38.2{ }^{\circ} \mathrm{C}$. In addition, two patients were febrile but the temperature was not recorded. Vomiting (42.3\%) was also commonly seen. Other symptoms included meningeal irritation, coma, epilepsy, hearing loss, blurred vision, and blindness. Sixteen patients had pulmonary cryptococcosis. Among them, 5 patients had cough, and 1 patient had shortness of breath. Respiratory symptoms were not observed in 11 other patients. Two patients with cryptococcal sinusitis presented with nasal congestion and pain.

\section{Laboratory findings}

Lumbar punctures at baseline were performed in all 33 patients. Most of the CNS infected patients had elevated opening pressure, CSF cell count and protein level, as well as decreased CSF glucose concentration. CSF culture was positive in 17 (85\%) out of 20 patients, while 23 out of 25 (92\%) had positive India ink smears for Cryptococcus. CSF cryptococcal antigen test results were documented in 20 patients, with all being positive and ranging from 1:640 to over 1:1280. Moreover, CSF parameters were normal in patients without CNS infection as summarized in Table 2. Among the 33 patients included, serum cryptococcal antigen titer was documented in 22 cases. Nineteen of them were positive, ranging from 1:10 to over 1:1280. Three patients, whose antigen titers in blood were negative, had a confirmed diagnosis of pulmonary cryptococcosis through histopathology. Serum titers equal or greater than 1:640 were observed more frequently in CNS infected patients than 
Table 1 Summary of clinical characteristics of 33 cryptococcosis patients with hematological diseases

\begin{tabular}{|c|c|}
\hline Variables & No. (\%) of patients $(N=33)$ \\
\hline Age, years & 50 (range, 16-79) \\
\hline Sex, female & 19 (57.6) \\
\hline \multicolumn{2}{|l|}{ Type of hematological diseases } \\
\hline Hematological malignancies & $12(36.4)$ \\
\hline $\mathrm{NHL}$ & $4(12.1)$ \\
\hline WM & $3(9.1)$ \\
\hline ALL & $1(3.0)$ \\
\hline$C L L$ & $1(3.0)$ \\
\hline $\mathrm{HL}$ & $1(3.0)$ \\
\hline MM & $1(3.0)$ \\
\hline MDS & $1(3.0)$ \\
\hline Non-malignant hematological disorders & $21(63.6)$ \\
\hline AlHA & $10(30.3)$ \\
\hline Evans' syndrome & $6(18.2)$ \\
\hline ITP & $5(15.1)$ \\
\hline \multicolumn{2}{|l|}{ Other underlying conditions } \\
\hline Chronic kidney diseases & $2(6.1)$ \\
\hline Splenectomy & $2(6.1)$ \\
\hline Rectal cancer & $1(3.0)$ \\
\hline Chemotherapy & $8(24.2)$ \\
\hline Glucocorticoids administration & $28(84.8)$ \\
\hline \multicolumn{2}{|l|}{ Sites of cryptococcal infection ${ }^{a}$} \\
\hline Brain & $26(78.8)$ \\
\hline Lung & $16(48.5)$ \\
\hline Blood & $3(9.1)$ \\
\hline Sinus & $2(6.1)$ \\
\hline
\end{tabular}

NHL non-Hodgkin's lymphoma; WM Waldenstrom's macroglobulinemia; ALL acute lymphocytic leukemia; CLL chronic lymphocytic leukemia; HL Hodgkin's lymphoma; MM multiple myeloma; MDS myelodysplastic syndrome; AIHA autoimmune hemolytic anemia; Evans' Evans' syndrome; ITP immune thrombocytopenia

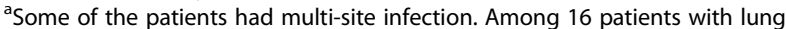
infection, 9 had brain involvement as well. All patients with blood or sinus involvement had brain or lung infection at the same time

those without CNS infection (15/15 vs. $1 / 7, \chi^{2}=13.866$, $P<0.001)$. Blood culture analyzed in 12 patients for Cryptococcus showed 3 to be positive (25\%).

\section{Image findings}

Abnormal cranial findings were presented in 18 out of 25 (72\%) meningitis patients either by CT scan or magnetic resonance imaging (MRI). Common sites of lesions were frontal lobe in 7, parietal lobe in 5 , basal ganglion in 6 , periventricular region in 6 , temporal lobe in 2 , occipital lobe in 2 , and cerebellum in 1 patient. Some patients had multisite involvement. Lesions were characterized by decreased density in the CT scan. MRI usually revealed low signal in $\mathrm{T} 1$ and high signal in T2, with or without enhancement.
Abnormalities of chest CT were found in all 16 patients with pulmonary infections. The most common findings were single or multi nodules or masses (14/16). Patchy airspace consolidation and cavities were described in 7 and 2 patients respectively. Pleural effusion was only documented in one patient. Some patients had more than two types of lesions on the chest CT scan.

\section{Treatment and outcomes}

All patients received antifungal treatment. Among the 26 cryptococcal meningitis patients, 18 were initially treated with amphotericin B (AmB) based therapy. Amphotericin B deoxycholate (d-AmB) daily dosage ranged from 20 to $30 \mathrm{mg}(0.35-0.59 \mathrm{mg} / \mathrm{kg} / \mathrm{d}$, median $0.43 \mathrm{mg} / \mathrm{kg} / \mathrm{d})$. Median of accumulated d-AmB dosage was $1425 \mathrm{mg}$ and ranged from 106 to $7400 \mathrm{mg}$. Note that liposomal AmB (L-AmB) was used in 2 patients with a daily dosage of $50 \mathrm{mg}$, and total dose of $850 \mathrm{mg}$ and $1425 \mathrm{mg}$, respectively. As shown in Table 3, at the end of initial therapy 10 out of 18 (55.6\%) patients achieved clinical success (all were partial response), 4 (22.2\%) patients had stable response, one patient had progression of disease and 2 patients died. Fluconazole-based initial therapy was administered to 5 CNS-infected patients. One patient died on the first day of antifungal treatment and was not included in the efficacy assessment. None of the remaining 4 patients achieved clinical success (all showed stable response). Three more patients received initial therapy with voriconazole or itraconazole, but only one patient showed a partial response. Three patients were given an additional $\mathrm{d}$-AmB intrathecal injection. Surgical intervention was performed in 2 patients, one with external ventricular drainage and another with ventriculoperitoneal shunt. The median duration of total antifungal therapy among these 26 patients was 18 weeks, ranging from one day to 160 weeks. Three patients died within the first 2 weeks of antifungal therapy $(3 / 26,11.6 \%)$ and five patients died within 10 weeks $(5 / 25,20.0 \%)$. Survival rate at 1-year follow-up was $72.0 \%(18 / 25)$. One patient each were lost in the 10-week and 1-year follow-up. These data are summarized in Table 3.

For patients without CNS involvement, 5 patients were treated with fluconazole, while the other two were treated with $\mathrm{d}-\mathrm{AmB}$ as THE initial therapy (total dosage of $500 \mathrm{mg}$ ). The median time of antifungal therapy duration was also 18 weeks and ranged from 3 to 27 weeks. Clinical response for pulmonary cryptococcosis was $85.7 \%(6 / 7)$. Pulmonary lobectomy was performed in one patient. Only one patient died of hepatic failure (drug-unrelated) after 4 weeks of fluconazole-based therapy. In pulmonary cryptococcosis patients, 2-week and 10-week mortality was $0 \%$ and $14.3 \%$, respectively. One-year survival rate was $85.7 \%$.

Twelve out of 18 CNS-infected patients who received AmB had drug-related ADEs, including hypokalemia (4 certain cases), thrombocytopenia (4 cases with 2 certain, 1 
Table 2 Clinical manifestations and laboratory findings of 33 patients

\begin{tabular}{|c|c|c|}
\hline Variables & Patients with CNS involvement $(N=26)$ & Patients without CNS involvement $(N=7)$ \\
\hline \multicolumn{3}{|l|}{ Manifestations, n (\%) } \\
\hline Fever & $24(92.3)$ & $1(14.3)$ \\
\hline Headache & $24(92.3)$ & 0 \\
\hline Vomiting & $11(42.3)$ & 0 \\
\hline Meningeal irritation signs & $8(30.8)$ & 0 \\
\hline Coma & $7(26.9)$ & 0 \\
\hline Epilepsy & $7(26.9)$ & 0 \\
\hline Hearing loss & $3(11.5)$ & 0 \\
\hline Blurred vision & $2(7.7)$ & 0 \\
\hline Cough & $2(7.7)$ & $3(42.9)$ \\
\hline Shortness of breath & 0 & $1(14.3)$ \\
\hline Asymptomatic & 0 & $4(57.1)$ \\
\hline Other symptoms ${ }^{a}$ & $3(11.5)$ & 0 \\
\hline \multicolumn{3}{|l|}{ Baseline CSF parameters } \\
\hline Opening Pressure, $\mathrm{mmH} 2 \mathrm{O}^{\mathrm{b}}$ & $>300(120,>300)$ & $113(75,260)$ \\
\hline $\mathrm{WBC}$, cells $/ \mathrm{mm}^{3 \mathrm{c}}$ & $28(1,533)$ & $2(1,7)$ \\
\hline Protein, $g / L^{d}$ & $0.77(0.18,2.6)$ & $0.28(0.16,0.73)$ \\
\hline Glucose, $\mathrm{mmol} / \mathrm{L}^{\mathrm{e}}$ & $1.6(0.14,5.32)$ & $3.2(2.8,3.9)$ \\
\hline CrAg titer $^{f}$ & $1: 1280(1: 640,>1: 1280)$ & All negative \\
\hline Indian ink smear, + /- 9 & $23 / 2$ & $0 / 7$ \\
\hline Culture for Cryptococcus, $+/{ }^{\mathrm{h}}$ & $17 / 3$ & $0 / 7$ \\
\hline Baseline serum CrAg titer ${ }^{i}$ & $1: 1280(1: 640,1: 5120)$ & 1:10 (Negative, 1:640) \\
\hline
\end{tabular}

CNS central nervous system; CSF cerebrospinal fluid; WBC white blood cell count; CrAg cryptococcal antigen

${ }^{a}$ Other symptoms included blindness in 1, loss of recent memory in 1 and symptoms of brain stem herniation in 1

${ }^{b} \mathrm{CSF}$ opening pressure was not documented in 5 CNS infected patients and 2 CNS uninfected patients

${ }^{c}$ CSF WBC was not documented in 6 CNS infected patients

${ }^{\mathrm{d}}$ CSF protein was not documented in 4 CNS infected patients

${ }^{e}$ CSF glucose was not documented in 4 CNS infected patients

${ }^{f}$ CSF CrAg was not documented in 5 CNS infected patients. In 1 patient, CSF CrAg was positive, but titer was not recorded

${ }^{9} \mathrm{CSF}$ Indian ink smear was not documented in 1 CNS infected patient

${ }^{\text {h}}$ CSF culture for Cryptococcus was not documented in 6 CNS infected patients

'Serum CrAg titer was not documented in 11 CNS infected patients

probable and 1 possible), leukopenia (1 certain case), renal function abnormalities (1 certain case, 1 possible case), and liver function abnormalities (1 possible case). Three patients were switched from AmB-based therapy to other therapies; two of them due to thrombocytopenia, and the reason for the third patient was not documented. Flucytosine was added in 18 patients as part of the initial therapy. Two of them withdrew flucytosine due to allergy (possible) and leukopenia (possible), respectively. No ADEs were reported during fluconazole treatment.

\section{Comparison between patients with hematological malignancies and non-malignancies}

In total, there were 12 patients with hematological malignancies and 21 with non-malignancies (Table 4). The patients in these two groups showed no significant differences in age or gender. Chemotherapy was more frequently administered to patients with hematological malignancies
( $8 / 12$ vs. $0 / 21, P<0.001)$, while more patients with nonmalignancies received glucocorticoids (20/21 vs. 8/12, $P=0.047)$. Most non-malignant hematological patients (20/21, 95.2\%) had CNS infection. In contrast, only six (6/ $12,50.0 \%)$ patients with hematological malignancies had CNS involvement ( $\left.\chi^{2}=9.351, P=0.001\right)$.

In cryptococcal meningitis patients, clinical characteristics such as manifestation, laboratory and radiology findings, treatment and outcomes were also compared between cases with hematological malignancies and non-malignancies (Table 5). Vomiting was more common in the hematological non-malignancies group $(11 / 20$ vs. $0 / 6, P=0.024)$. However, no significant differences were found in other items.

\section{Discussion}

In previous studies, hematological neoplasis was the most common concomitant conditions in patients with hematological diseases, among which lymphoma was 
Table 3 Initial antifungal therapy and outcomes of 26 patients with CNS involvement

\begin{tabular}{ll}
\hline Variables & No. (\%) of patients $(N=26)$ \\
\hline Initial antifungal therapy & $18(69.2)$ \\
AmB based & $5(19.2)$ \\
Fluconazole based & $3(11.6)$ \\
Other ${ }^{\mathrm{a}}$ & \\
Efficacy of initial antifungal therapy & $11(42.3)$ \\
Success & 0 \\
Complete response & $11(42.3)$ \\
Partial response & $15(57.7)$ \\
Failure & $10(38.5)$ \\
Stable & $1(3.8)$ \\
Progression & $4(15.4)$ \\
Death & \\
Outcome & \\
2-week mortality rate & $3 / 26(11.6 \%)$ \\
10-week mortality rate & $5 / 25(20.0 \%)$ \\
1-year survival rate & $18 / 25(72.0 \%)$ \\
\hline
\end{tabular}

CNS central nervous system; $A m B$ amphotericin B

${ }^{a}$ Other initial antifungal therapies included voriconazole in 2 , itraconazole in 1 patient

${ }^{\mathrm{b}}$ One patient lost for 10-week and 1-year follow-up

most frequently reported [18-21]. Only one study showed that leukemia was the most common underlying hematological malignancy in cryptococcosis patients [22]. Our study reviewed 33 cryptococcosis cases predisposed to hematological diseases in a 14-year span and showed that lymphoma $(41.7 \%)$ was the most frequently encountered malignancy. Notably, only a few cases of cryptococcosis have been reported in patients with non-malignant hematological conditions. A recently published literature review summarized the clinical features of cryptococcal meningitis in 5 patients with AIHA and 8 previously reported other cases [11]. Additionally, in Pappas's large study on HIV-negative cryptococcosis, 29 out of 306 (9\%) subjects had concomitant hematological malignancies, while no cases with hematological non-malignant conditions were reported [23]. In Lee's 18-year study on cryptococcal meningitis, $21.6 \%$ had predisposing hematological and oncological conditions; however, a detailed breakdown was not given [24]. In our study, majority (21/33) of the patients had hematological non-malignant conditions. AIHA was the most common one, accounting for $47.6 \%$ of non-malignancies and followed by ITP and Evans' syndrome. Considering these data, it is likely that cryptococcosis may be underestimated among this subpopulation.

Brain, lung and blood were undoubtedly found to be the most common infection sites. The majority of cryptococcosis cases in our series had CNS involvement, which is much higher than the description by Tseng in non-HIV patients (53.7\%) and similar to that in HIV conditions (81.5\%) [25]. Clinical manifestations of patients were unspecific [26, 27]. The most common symptoms of CNS infection in our study were fever, headache, and vomiting. In addition, many of these patients had temperature over $38.5{ }^{\circ} \mathrm{C}$, which is consistent with a previous report that immunocompromised patients were more likely to have high fever [28]. Identification of the infecting pathogen was performed through CSF culture or India ink smear, which are traditional methods for the diagnosis of cryptococcal meningitis. Almost all of the CNS-infected patients (25/26) were confirmed by either positive culture or smear. In addition to these methods, cryptococcal antigen test is an important addition to the diagnostics and is widely used [29]. In

Table 4 Comparison of clinical characteristics between patients with hematological malignancies and non-malignancies $(N=33)$

\begin{tabular}{|c|c|c|c|}
\hline Variables & $\begin{array}{l}\text { Patients with hematological } \\
\text { malignancies (\%) } \\
(N=12)\end{array}$ & $\begin{array}{l}\text { Patients with hematological } \\
\text { non-malignancies (\%) } \\
(N=21)\end{array}$ & $P$ value \\
\hline Age, years & $52.5(16-79)$ & $50(20-77)$ & 0.586 \\
\hline Sex, female & $3(25.0)$ & $16(76.2)$ & 0.126 \\
\hline \multicolumn{4}{|c|}{ Non-hematological underlying conditions } \\
\hline Chronic kidney diseases & $0(0)$ & $2(9.5)$ & 0.523 \\
\hline Splenectomy & $0(0)$ & $2(9.5)$ & 0.523 \\
\hline Rectal cancer & $0(0)$ & $1(4.8)$ & 1.000 \\
\hline Chemotherapy & $8(66.7 \%)$ & $0(0)$ & $<0.0001$ \\
\hline Glucocorticoids administration & $8(66.7 \%)$ & $20(95.2 \%)$ & 0.047 \\
\hline \multicolumn{4}{|l|}{ Sites of cryptococcal infection } \\
\hline Brain & $6(50.0)$ & $20(95.2)$ & 0.001 \\
\hline Lung & $8(66.7)$ & $8(38.1)$ & 0.157 \\
\hline Blood & $1(8.3)$ & $2(9.5)$ & 1.000 \\
\hline Sinus & $1(8.3)$ & $1(4.8)$ & 1.000 \\
\hline
\end{tabular}


Table 5 Comparison of manifestations, laboratory finding, clinical response of antifungal treatment, outcomes between cryptococcal meningitis patients with hematological malignancies and non-malignancies

\begin{tabular}{|c|c|c|c|}
\hline Variables & $\begin{array}{l}\text { CM Patients with hematological } \\
\text { malignancies (\%) } \\
(N=6)\end{array}$ & $\begin{array}{l}\text { CM Patients with hematological } \\
\text { non-malignancies }(\%) \\
(N=20)\end{array}$ & $P$ value \\
\hline \multicolumn{4}{|l|}{ Symptoms } \\
\hline Fever & $5(83.3)$ & $19(95.0)$ & 0.415 \\
\hline Headache & $6(100.0)$ & $18(90.0)$ & 1.000 \\
\hline Vomitting & $0(0.0)$ & $11(55.0)$ & 0.024 \\
\hline \multicolumn{4}{|l|}{ Radiology of brain ${ }^{a}$} \\
\hline Multisite lesions, Yes/No & $5 / 1$ & $10 / 8$ & 0.351 \\
\hline Parenchyma damage, Yes/No & $5 / 1$ & $13 / 5$ & 1.000 \\
\hline \multicolumn{4}{|l|}{ Baseline CSF parameters } \\
\hline Opening Pressure $>250 \mathrm{mmH}_{2} \mathrm{O}, \mathrm{Yes} / \mathrm{No}^{\mathrm{b}}$ & $3 / 2$ & $10 / 6$ & 1.000 \\
\hline WBC, cells $/ \mathrm{mm}^{3 \mathrm{c}}$ & $31.5(20,40)$ & $34.5(1,533)$ & 0.802 \\
\hline Protein, $g / L^{d}$ & $0.74(0.184,1.015)$ & $0.74(0.277,2.601)$ & 0.773 \\
\hline Glucose $<1.1 \mathrm{mmol} / \mathrm{L}$, Yes/No ${ }^{\mathrm{e}}$ & $4 / 1$ & $13 / 4$ & 1.000 \\
\hline Indian ink smear, $+/-^{f}$ & $6 / 0$ & $17 / 2$ & 1.000 \\
\hline Culture for Cryptococcus, $+/{ }^{\mathrm{g}}$ & $3 / 1$ & $14 / 2$ & 0.509 \\
\hline CrAg titer $>1: 1280$, Yes $/ \mathrm{No}^{\mathrm{h}}$ & $1 / 5$ & $5 / 9$ & 0.613 \\
\hline Baseline serum CrAg titer >1:1280, Yes/ $\mathrm{No}^{\mathrm{i}}$ & $4 / 2$ & $5 / 8$ & 0.350 \\
\hline \multicolumn{4}{|l|}{ Treatment } \\
\hline AmB-based therapy & $4(66.7)$ & $14(70.0)$ & 1.000 \\
\hline Success & $3(50.0)$ & $8(40.0)$ & 1.000 \\
\hline \multicolumn{4}{|l|}{ Outcome } \\
\hline 2-week mortality & $0(0.0)$ & $3(15.0)$ & 1.000 \\
\hline 10-week mortality ${ }^{j}$ & $1(16.7)$ & $4(21.1)$ & 1.000 \\
\hline
\end{tabular}

amage findings of the brain was not documented in 2 patients with hematological non-malignancies

${ }^{\mathrm{b} C S F}$ opening pressure was not documented in 1 patient with hematological malignancies and 4 with non-malignancies

${ }^{c}$ CSF WBC was not documented in 2 patient with hematological malignancies and 4 with non-malignancies

${ }^{d}$ CSF protein was not documented in 1 patient with hematological malignancies and 3 with non-malignancies

${ }^{\text {e}} \mathrm{CSF}$ glucose was not documented in 1 patient with hematological malignancies and 3 with non-malignancies

${ }^{f} \mathrm{CSF}$ Indian ink smear was not documented in 1 patient with hematological non-malignancies

${ }^{9} \mathrm{CSF}$ culture for Cryptococcus was not documented in 2 patient with hematological malignancies and 4 with non-malignancies

${ }^{\mathrm{h}} \mathrm{CSF}$ CrAg was not documented in 6 patients with hematological non-malignancies

iSerum CrAg titer was not documented in 7 patients with hematological non-malignancies

jone patient with hematological non-malignancies was lost for 10-week follow-up

cryptococcal meningitis patients, the positivity of CSF cryptococcal antigen test could be up to $100 \%$, indicating that this test should be conducted in suspected CNSinvolved patients as well as the CSF culture and smear. Patients with only lung involvement were either asymptomatic, or only had mild cough. Abnormal lesions in the lung can be the only clue for cryptococcal infection. This would make early diagnosis of these cases more difficult, and perhaps may increase the possibility of CNS dissemination. Thus, cryptococcal antigen test of serum are needed for differential diagnosis. In this study, we observed that the serum cryptococcal antigen titer in CNS-infected patients was relatively higher (equal or higher than 1:640). This is consistent with that in HIV-infected conditions more than that in HIV-uninfected ones [25]. In patients with only pulmonary involvement, 4 were positive for cryptococcal antigen in blood and 3 were negative. The proportion with higher titers in blood was lower in these patients than that in cryptococcal meningitis. Similar findings were reported in a previous study, which showed that infection in the lung resulted in lower cryptococcal antigen and a higher incidence of negative serum antigen tests [26]. As a result, lung biopsy should be conducted when pulmonary cryptococcosis is suspected with a negative antigen test; indeed, we confirmed the pulmonary cryptococcosis diagnosis in 3 of our patients using this method.

Treatment of cryptococcal meningitis in the setting of hematological diseases is difficult, due to the impaired immunity, frequent ADEs, and the lack of supporting evidence for this small population. The Infectious Diseases 
Society of America (IDSA) guideline provides a general recommendation of $\mathrm{d}-\mathrm{AmB}$ with or without flucytosine therapy for non-HIV patients however, the regimen for patients with hematological disorders was not specified [30]. Based on recently published guideline for CNS infections in patients with hematological disorders (including hematological malignancies and allogeneic stem-cell transplantation), L-AmB or AmB lipid complex (ABLC) should be the first choice for cryptococcal meningitis as drug related bone marrow suppression and hemolysis are of great concern [31]. In our case series, most patients were treated with d-AmB-based initial therapy according to the Chinese guideline for cryptococcosis at a dosage of $0.35-0.59 \mathrm{mg} /$ $\mathrm{kg} / \mathrm{d}$ (median $0.43 \mathrm{mg} / \mathrm{kg} / \mathrm{d}$ ), and the majority of them also received flucytosine $(100 \mathrm{mg} / \mathrm{kg} / \mathrm{d})$. Clinical responses were achieved in most of them, consistent with our previous study in HIV-uninfected patients with cryptococcal meningitis [28]. Both d-AmB and flucytosine were well tolerated in our series, suggesting that $\mathrm{d}-\mathrm{AmB}$ with or without flucytosine could be a safe and effective choice for treatment of cryptococcal meningitis in hematological patients. For patients without CNS infection, fluconazole with or without flucytosine was used. In this group, most patients tolerated fluconazole well and had a favorable response.

Previous studies on cryptococcal infection in hematological settings in general have emphasized the malignant conditions. However, we observed that nonmalignant hematological patients seemed more likely to develop CNS infection than malignancy patients. The high proportion of long-term corticosteroids administration in non-malignant patients, which could lead to infection dissemination, may in part, be responsible for this difference. Furthermore, in the fatal cryptococcal meningitis, the two groups of patients did not differ significantly in other baseline characteristics such as symptoms, laboratory findings, treatment response, and outcomes. Therefore, cryptococcal infection, especially CNS involvement should not be neglected in hematological non-malignant patients.

To our knowledge, this is the largest case series of cryptococcosis patients with hematological diseases to date. However, our study has several limitations. It is a single center-based study in a tertiary care hospital, so that the number of cases we enrolled was small. All cases in our cohort were HIV-uninfected; thus, our experience may not be representative of those with HIVinfection. Meanwhile, due to the retrospective nature of our study design, some of the clinical data are incomplete, which decreased the power of statistical analysis. Larger, multicenter studies are needed to fully identify the characteristics of this population. Moreover, randomized controlled trials should be conducted to compare the different antifungal regimens to provide specific evidence for clinical practice in the future.

\section{Conclusions}

Cryptococcal infection has been known to be uncommon in patients with hematological diseases and thus can be easily misdiagnosed. In addition to commonly identified hematological malignant conditions, non-malignant conditions are also risk factors for cryptococcal infection and have a tendency for CNS involvement. Symptoms of cryptococcal infection in hematological patients were similar with other patient groups and were unspecific, suggesting the importance of cryptococcal antigen test, and CSF culture or smear. D-AmB based initial treatment could be a choice for cryptococcal meningitis patients with hematological diseases, while most patients with only lung involvement could be treated with fluconazole.

\section{Additional file}

Additional file 1: Table S1. Detailed clinical characteristics of 33 cryptococcosis patients with hematological diseases. (DOC 86 kb)

\section{Abbreviations}

ABLC: amphotericin B lipid complex; ADEs: adverse drug events; AlHA: autoimmune hemolytic anemia; ALL: acute lymphocytic leukemia; AmB: amphotericin B; CLL: chronic lymphocytic leukemia; CNS: central nervous system; CSF: cerebrospinal fluid; CT: computerized tomography; d-AmB: amphotericin B deoxycholate; EORTC/MSG: Mycoses Study Group and European Organization for Research and Treatment of Cancer; HIV: human immunodeficiency virus; HL: Hodgkin's lymphoma; HSCT: hematopoietic stem cell transplantation; ICD: International Classification of Diseases; IDSA: Infectious Diseases Society of America; ITP: immune thrombocytopenia; L-AmB: liposomal amphotericin B; MDS: myelodysplastic syndrome; MM: multiple myeloma; MRI: magnetic resonance imaging; NHL: non-Hodgkin's lymphoma; WBC: white blood cell; WHO-UMC: World Health Organization Collaborating Centre for International Drug Monitoring, the Uppsala Monitoring Centre; WM: Waldenstrom's macroglobulinemia

\section{Acknowledgements}

We thank all the health care workers who took care of these patients. And we would like to thank the staff in Medical Records Room, Huashan Hospital, who kept these valuable discharge data.

\section{Funding}

Not applicable.

\section{Availability of data and materials}

The datasets used and analyzed in this study are available from the corresponding author on reasonable request.

\section{Authors' contributions}

LPZ designed this study. YHC and HZZ collected the data from medical records. RYW, YQC, JQW and XW analyzed and interpreted the data. RYW and YQC were equal contributors in writing the manuscript. All authors read and approved the final manuscript.

\section{Ethics approval and consent to participate}

This study was reviewed and approved by the local medical Ethics Committee of Huashan Hospital, Fudan University, Shanghai, China. As this is a retrospective study, data was obtained through medical records and analyzed anonymously, and therefore informed consent of the participants was not required.

Consent for publication

Not applicable. 


\section{Competing interests}

The authors declare that they have no competing interests.

\section{Publisher's Note}

Springer Nature remains neutral with regard to jurisdictional claims in published maps and institutional affiliations.

Received: 21 December 2016 Accepted: 22 June 2017 Published online: 03 July 2017

\section{References}

1. Pyrgos V, Seitz AE, Steiner CA, Prevots DR, Williamson PR. Epidemiology of cryptococcal meningitis in the US: 1997-2009. PLoS One. 2013;8:e56269.

2. Sloan DJ, Parris V. Cryptococcal meningitis: epidemiology and therapeutic options. Clin Epidemiol. 2014;6:169-82.

3. Park BJ, Wannemuehler KA, Marston BJ, Govender N, Pappas PG, Chiller TM. Estimation of the current global burden of cryptococcal meningitis among persons living with HIV/AIDS. AIDS. 2009;23:525-30.

4. Husain S, Wagener MM, Singh N. Cryptococcus neoformans Infection in organ transplant recipients: variables influencing clinical characteristics and outcome. Emerg Infect Dis. 2001;7:375-81

5. Neofytos D, Fishman JA, Horn D, et al. Epidemiology and outcome of invasive fungal infections in solid organ transplant recipients. Transpl Infect Dis. 2010;12:220-9.

6. Lewis RE, Cahyame-Zuniga L, Leventakos K, et al. Epidemiology and sites of involvement of invasive fungal infections in patients with haematological malignancies: a 20-year autopsy study. Mycoses. 2013;56:638-45.

7. Neofytos D, Horn D, Anaissie E, et al. Epidemiology and outcome of invasive fungal infection in adult hematopoietic stem cell transplant recipients: analysis of multicenter prospective antifungal therapy (PATH) alliance registry. Clin Infect Dis. 2009;48:265-73.

8. Kontoyiannis DP, Marr KA, Park BJ, et al. Prospective surveillance for invasive fungal infections in hematopoietic stem cell transplant recipients, 20012006: overview of the transplant-associated infection surveillance network (TRANSNET) database. Clin Infect Dis. 2010;50:1091-100.

9. Pagano L, Caira M, Candoni A, et al. The epidemiology of fungal infections in patients with hematologic malignancies: the SEIFEM-2004 study. Haematologica. 2006;91:1068-75.

10. Donhuijsen K, Petersen P, Schmid WK. Trend reversal in the frequency of mycoses in hematologic neoplasias: autopsy results from 1976 to 2005. Dtsch Arztebl Int. 2008;105:501-6.

11. Yang $Y$, Sang J, Pan W, et al. Cryptococcal meningitis in patients with autoimmune hemolytic anemia. Mycopathologia. 2014;178:63-70.

12. Hu XP, Wang RY, Wang $X$, et al. Dectin-2 polymorphism associated with pulmonary cryptococcosis in HIV-uninfected Chinese patients. Mycoses. 2015:53(8):810.

13. De PB, Walsh TJ, Donnelly JP, et al. Revised definitions of invasive fungal disease from the European Organization for Research and Treatment of cancer/invasive fungal infections cooperative group and the National Institute of Allergy and Infectious Diseases mycoses study group (EORTC/ MSG) co. Clin Infect Dis. 2008;46(12):1813-21.

14. Weng $\mathrm{XH}$, Zhu LP, Wen $\mathrm{H}$, et al. Clinical practioce onsensus on the management of cryptococcal infection. Chin J Mycol. 2010;5:65-8.

15. Yan D, Huang JR, Lian JS, et al. Treatment of cryptococcal meningitis with low-dose amphotericin B and flucytosine. Chin Med J. 2012;125:385-7.

16. Segal BH, Herbrecht $R$, Stevens DA, et al. Defining responses to therapy and study outcomes in clinical trials of invasive fungal diseases: mycoses study group and European Organization for Research and Treatment of Cancer Consensus criteria. Clin Infect Dis. 2008;47:674-83.

17. The use of the WHO-UMC system for standardised case causality assessment (last updated January 2013). https://www.who-umc.org/media/ 2768/standardised-case-causality-assessment.pdf. Accessed 1 Feb 2015.

18. Khodunova EE, Frolova IN, Parovichnikova EN, et al. Cryptococcosis in hematology practice. Ter Arkh. 2013;85:41-6.

19. Kontoyiannis DP, Peitsch WK, Reddy BT, et al. Cryptococcosis in patients with cancer. Clin Infect Dis. 2001:32:e145-50.

20. Kaplan MH, Rosen PP, Armstrong D. Cryptococcosis in a cancer hospital: clinical and pathological correlates in forty-six patients. Cancer. 1977:39: 2265-74.

21. Shih CC, Chen YC, Chang SC, Luh KT, Hsieh WC. Cryptococcal meningitis in non- HIV-infected patients. QJM. 2000;93:245-51.
22. Pagano L, Fianchi L, Caramatti C, et al. Cryptococcosis in patients with hematologic malignancies. A report from GIMEMA-infection. Haematologica. 2004;89:852-6

23. Pappas PG, Perfect JR, Cloud GA, et al. Cryptococcosis in human immunodeficiency virus-negative patients in the era of effective azole therapy. Clin Infect Dis. 2001;33:690-9.

24. Lee YC, Wang JT, Sun HY, Chen YC. Comparisons of clinical features and mortality of cryptococcal meningitis between patients with and without human immunodeficiency virus infection. J Microbiol Immunol Infect. 2011; 44:338-45.

25. Tseng HK, Liu CP, Ho MW, et al. Microbiological, epidemiological, and clinical characteristics and outcomes of patients with cryptococcosis in Taiwan, 1997-2010. PLoS One. 2013;8:e61921.

26. Baddley JW, Perfect JR, Oster RA, et al. Pulmonary cryptococcosis in patients without HIV infection: factors associated with disseminated disease. Eur J Clin Microbiol. 2008;27:937-43.

27. Bicanic T, Harrison TS. Cryptococcal meningitis. Br Med Bull. 2004;72(1):99-118.

28. Zhu LP, Wu JQ, Xu B, Ou XT, Zhang QQ, Weng XH. Cryptococcal meningitis in non-HIV-infected patients in a Chinese tertiary care hospital, 1997-2007. Med Mycol. 2010;48(4):570-9.

29. Jarvis JN, Harrison TS, Lawn SD, et al. Cost effectiveness of cryptococcal antigen screening as a strategy to prevent HIV-associated cryptococcal meningitis in South Africa. PLoS One. 2013;8:e69288.

30. Perfect JR, Dismukes WE, Dromer F, et al. Clinical practice guidelines for the management of cryptococcal disease: 2010 update by the Infectious Diseases Society of America. Clin Infect Dis. 2010;50(3):291-322.

31. Schmidthieber M, Silling G, Schalk $E$, et al. CNS infections in patients with hematological disorders (including allogeneic stem cell transplantation) guidelines of the Infectious diseases working party (AGIHO) of the German Society of Hematology and Medical Oncology (DGHO). Ann Oncol. 2016; 27(7):1207-25

\section{Submit your next manuscript to BioMed Central and we will help you at every step:}

- We accept pre-submission inquiries

- Our selector tool helps you to find the most relevant journal

- We provide round the clock customer support

- Convenient online submission

- Thorough peer review

- Inclusion in PubMed and all major indexing services

- Maximum visibility for your research

Submit your manuscript at www.biomedcentral.com/submit
) Biomed Central 